using the Systemic Lupus Erythematosus Activity Questionnaire (SLAQ). Multiple significant correlations of fatigue could be demonstrated with age, gender, sleep disorders, pain, physical activity, disease activity, obesity, NSAIDs intake, physical and psychological well-being, overall health status and restrictions on everyday life. The 5 strongest associations with fatigue are given in the table.

Based on these results, further research should be conducted to analyse the mentioned factors in more detail. It remains an interesting hypothesis that therapy aimed to improve one or more associated factors identified will also improve patients' reported fatigue.

\section{PS8:159 MOVING WITH THE TIMES: SOCIAL MEDIA USE AMONGST LUPUS PATIENTS}

LM Wheeler, A Pakozdi, R Rajakariar, M Lewis, A Cove-Smith, D Pyne. Barts Lupus Centre, Barts Health NHS Trust, London, UK

\subsection{6/lupus-2018-abstract.202}

Background Over the last decade the rise in social media use has been almost exponential. There are numerous online platforms where patients can interact for support and information gathering. A study presented at the ACR in 2013 showed lupus patients were willing to participate in Facebook chats for the purposes of disease education. ${ }^{1}$ We sought to discover current usage of social media amongst an ethnically mixed population attending an inner city tertiary lupus centre.

Methods Questionnaires were distributed to consecutive patients attending the Barts Lupus Centre from October 2016 to February 2017. 17 questions asked about patient demographics and patients use of online information and support services, particularly social media platforms, with regards to lupus.

Results 84 completed questionnaires were returned. 83\% respondents were female. There were 28 South Asians, 26 Whites and 24 Blacks, 2 of other ethnic groups and 2 of mixed race. $64 \%(n=54)$ of patients accessed online lupus information and support sites. $45 \%$ percent $(n=38)$ of patients reported using social media sites (26\% South Asians, 34\% Blacks and $34 \%$ Whites). Of those using social media $22 \%$ $(n=8)$ patients used these tools daily and 30\% $(n=11)$ reported weekly use. Facebook $(n=20)$, blogs $(n=9)$, youtube $(n=9)$, and Instagram $(n=7)$ were cited as the most frequently used applications. Most patients $(n=30)$ sought information on the disease, $17(45 \%)$ wanted to find out about new treatments for lupus, 16 (42\%) sought new ways to self-manage their disease, 14 (37\%) sought interactions with other patients, and $10(26 \%)$ were seeking support online. Patients most commonly wanted information on skin and joint complaints and family planning. 66\% $(n=56)$ thought their rheumatology team should have an online social media application to communicate with their patients.

Conclusions A significant proportion of our lupus patients (45\%) use social media to access information and support for their disease. Facebook, blogs, Instagram and Youtube are commonly used. Social media applications can provide physicians with a tool to interact with lupus patients to improve accessibility to health care and better health outcomes.

\section{REFERENCE}

1. Spring NH. 2013 ACR/ARHP Annual Meeting, abstract 990.

\section{PS8:160 HEALTH CARE RESOURCE USE (HRU) AND MEDICAL COST ANALYSES AS A FUNCTION OF SYSTEMIC LUPUS ERYTHEMATOSUS (SLE) DISEASE SEVERITY: ANALYSIS OF CLAIMS DATA OF A GERMAN SICKNESS FUND}

${ }^{1}$ ER Hammond, ${ }^{2} \mathrm{H}$ Freidel, ${ }^{2} \mathrm{E}$ Garal-Pantaler, ${ }^{3} \mathrm{M}$ Pignot, ${ }^{4} \mathrm{E}$ Velthuis, ${ }^{1} \mathrm{X}$ Wang, ${ }^{5} \mathrm{H}$ Nab, ${ }^{1} \mathrm{~B}$ Desta, ${ }^{6} \mathrm{~A}$ Schwarting. ${ }^{1}$ AstraZeneca, Gaithersburg, MD, USA; ${ }^{2}$ Team Gesundheit GmbH, Essen, Germany; ${ }^{3}$ Kantar Health GmbH, Munich, Germany; ${ }^{4}$ Evidera PPD, Utrecht Area, The Netherlands; ${ }^{5}$ AstraZeneca, Cambridge, UK; ${ }^{6}$ Universitätsmedizin der Johannes GutenbergUniversität Mainz, Germany

\subsection{6/lupus-2018-abstract.203}

Purpose Real-world HRU and medical cost analyses support drug development in SLE. The economic burden of SLE as a function of disease severity was quantified for a German cohort.

Methods HRU and cost analyses were performed for patients ( $\geq 18$ years old) with SLE and compared with those of matched controls for 2009-2014 using the Betriebskrankenkassen (BKK) German Sickness Fund Database. BKK is a branch of the statutory German health insurance. SLE was confirmed in 2009 using the diagnosing physician's specialty, repeated SLE-related claims, co-diagnosis codes, laboratory tests, or prescriptions. At least 3 years' data was also required prior to 2009. SLE cases were control matched by age, sex, and baseline Charlson comorbidity index (CCI). Continuous outcomes were compared with nonparametric tests.

Results 1160 patients (mean age: 52 years; median baseline CCI: 2 [females], 3 [males]; baseline CCI range: 1-13) with SLE met all inclusion criteria. Most patients were female (84\%) and were diagnosed with SLE before 2009 (85\%). In 2009, SLE prevalence was $37.32 / 100,000$ and incidence was 5.96/100,000. Prevalence increased progressively to 47.36 100,000 in 2014. SLE disease severity was characterised as mild, moderate, and severe for 148, 484, and 528 patients, respectively, per ICD-10-GM and medication/procedures codes. Patients with moderate and severe SLE had greater mean annual medical costs in 2009-2014 than matched controls (e. g., in 2009, moderate SLE: $€ 4867$ vs $€ 3,380, \mathrm{p}<0.0001$; severe SLE: $€ 10001$ vs $€ 4,239$, p<0.0001). Mean costs and number of outpatient visits, hospital stays, outpatient prescriptions and other benefits, and total number of hospital days were significantly greater for the full SLE population and moderate and severe SLE subpopulations than for matched controls. For example, mean costs for hospital stays, outpatient prescriptions, and other benefits in 2009 were $€ 4335$ vs $€ 1,414$, $€ 2582$ vs $€ 1,087$, and $€ 1068$ vs $€ 691$, respectively, for patients with severe SLE vs controls.

Conclusions Economic burden was greater for patients with moderate to severe SLE than for matched controls. Patients with SLE had greater HRU and costs than matched controls. HRU increased with increasing SLE disease severity. New therapeutics that decrease disease activity could reduce economic burden.

\section{PS8:161 THE DISEASE BURDEN IN PATIENTS WITH LONGSTANDING SYSTEMIC LUPUS ERYTHEMATOSUS: FOCUS ON HEALTH RESOURCE USE AND COSTS}

${ }^{1} \mathrm{C}$ Tani, ${ }^{2} \mathrm{~V}$ Lorenzoni, ${ }^{1} \mathrm{R}$ Vagelli, ${ }^{1} \mathrm{C}$ Stagnaro, ${ }^{1} \mathrm{E}$ Elefante, ${ }^{1} \mathrm{~L}$ Carli, ${ }^{2} \mathrm{G}$ Turchetti, ${ }^{1} \mathrm{M}$ Mosca. ${ }^{1}$ Rheumatology Unit, University of Pisa, Italy; ${ }^{2}$ Management Institute, Sant'Anna - Scuola Universitaria Superiore, Pisa, Italy

10.1136/lupus-2018-abstract.204 